Published in final edited form as:

Transpl Int. 1988 July ; 1(2): 80-85.

\title{
Renal artery reconstruction for harvesting injuries in kidney transplantation with particular reference to the use of vascular allografts
}

\author{
Andreas G. Tzakis ${ }^{1}$, Vincenzo Mazzaferro ${ }^{1}$, Chen-En Pan ${ }^{1}$, Robert D.Gordon ${ }^{1}$, Satoru \\ Todo $^{1}$, Leonard Makowka ${ }^{1}$, and Thomas E.Starzl ${ }^{1,2}$ \\ ${ }^{1}$ Department of Surgery, University Health Center of Pittsburgh, University of Pittsburgh, 3601 Fifth \\ Avenue, Falk Clinic 4 West, Pittsburgh, PA 15213, USA \\ 2 Veterans Administration Medical Center, University Drive C, Pittsburgh, PA 15240, USA
}

\begin{abstract}
At the University of Pittsburgh during the calendar year 1986, an arterial injury occurred during harvesting in 20 (7.5\%) of the 270 grafts used to perform kidney transplantation (KTx). Four cases required reconstruction, using extension iliac arterial allografts from cadaveric donors of the same blood type; 6 patients, remodelling of the aortic patch in multiple arteries; 4 cases, repairs for injuries to the smaller segmental/polar arteries; 6 cases, a combination of the above techniques. These ex vivo arterial reconstructions are described and the use of donor arterial homografts is emphasized. No deaths have occurred at an average follow-up of 19 months. The postoperative acute tubular necrosis (ATN) rate was significantly higher ( $90 \%$ ) compared with non-reconstructed kidneys during the same year (30\%). The 1-year graft survival of kidneys undergoing arterial reconstruction (75\%) was statistically no different than the overall kidney-transplant survival. Whenever extension iliac allografts were utilized, the cyclosporin-steroid immunosuppression required to control the kidney rejection contributed to the long-term patency rate of the graft. Since the number of organs available for grafting is limited, reconstruction of injured renal vessels has become justified, allowing valuable kidneys to be used that would otherwise be lost.
\end{abstract}

\section{Keywords}

Arterial injury; Renal artery reconstruction; Kidney transplant

\begin{abstract}
Kidney transplantation (KTx) has become the treatment of choice for end-stage renal disease. Because the number of organs available for grafting is limited, we have used kidneys that required simple or extensive vascular reconstruction in order to be transplantable. Previously, such organs had quite often been discarded. We report our own experience with reconstruction of the renal artery for cadaveric kidney transplantation, including the use of cadaveric iliac arterial grafts, during the calendar year 1986.
\end{abstract}

\section{Materials and method}

Case material

During 1986 at the University of Pittsburgh, 270 cadaveric renal transplantations were performed under cyclosporin-steroid immunosuppression, to which OKT3 therapy was added 
when intractable rejection supervened. The blood flow to the kidney was monitored postoperatively with a technetium renal scintigram.

Twenty $(7.5 \%)$ of the 270 grafts required arterial reconstruction. Eight of the grafts with arterial reconstruction were procured locally by our own surgeons; the other 12 were shipped in from other centers.

Eighteen of the 20 donors were adults averaging $22 \pm 15$ (SD) years. Two pediatric donors were 6 and 8 years of age, respectively. Likewise, 18 of the 20 recipients were over 10 years old, averaging $30.4 \pm 13$ (SD) years; the only exceptions were two children 7 and 8 years of age, who received the two pediatric kidneys.

\section{Source of vascular allografts}

Iliac artery and vein grafts are taken at the time of multiple organ harvesting $[9,10]$, usually in continuity with the terminal aorta and inferior vena cava. The grafts are preserved in Terasaki tissue-culture medium. Grafts are discarded after 7 days of storage. In 11 of the 20 cases, the vascular grafts used were from the same donor as the kidney. Moreover, the grafts were always of the same blood type as the recipient.

\section{Types of repair}

Injury of the arterial supply to the cadaveric kidney can happen at various levels, and any segment can be involved. Such injuries, although serious, can usually be repaired with careful planning and microsurgical techniques. The techniques can be summarized as follows.

Type 1: extension iliac artery grafts-A transected and shortened single renal artery can usually be reconstructed with an end-to-end anastomosis to the internal iliac artery of the recipient $[4,8,9]$. Alternatively, a free external or common iliac artery graft can be anastomosed end-to-end to the damaged renal artery during the back table preparation. If the anastomosis is end-to-side to the external or common iliac artery, bifurcation of the free graft can be fashioned to provide a Carrel patch for an easier end-to-side anastomosis (Fig. 1). This is a particularly important option for older patients whose vessels are atherosclerotic.

Type 2: revision of aortic patch-This technique is useful in kidneys with a dual blood supply in which an injury of the aorta has occurred between the take-off of the renal arteries, or where the aortic take-off between the two arteries is separated by more than $1 \mathrm{~cm}$ (Fig. 2). Care must be taken to avoid tension or kinking of the arteries or of the renal vein.

Type 3: repair of injuries of smaller arteries-Small upper-polar artery injuries result in small infarcts at the upper pole of the graft, which are not harmful enough to warrant tedious efforts at vascular repair. Lower polar arterial injuries are potentially more dangerous because the blood supply to the ureter of the graft may be compromised. The lower polar vessels are repaired either directly (Fig. 3a), by reimplantation into a larger renal vessel (Fig. 3b), or by using a side-to-side "conjoined" anastomosis [5] in order to create a common ostium that can be anastomosed end-to-end to the hypogastric artery (Fig. 4a) or to a donor vascular graft (Fig. $4 b)$. The conjoined technique is possible only when the vessels are of sufficient length and similar size.

Type 4: combinations of types 1-3-All of the above-mentioned basic techniques can be used in combination (Figs. 5, 6). One type-4 patient had the most extensive reconstruction known to have been performed in any renal recipient. This was a 55-year-old male with endstage renal disease and severe peripheral vascular disease; he was explored for kidney transplantation. Renal transplantation was not possible without replacement of his calcified 
and friable infrarenal aorta and iliac vessels. An aortoiliac allograft from the renal cadaveric donor was used, as shown in Fig. 6, as well as iliac grafts from another donor of the same blood type to bridge the right iliac system of the recipient. In addition, a right femoropopliteal bypass with a reversed autologous saphenous vein graft was needed distally. Postoperatively, the patient developed ischemic colitis, which required sigmoid resection and colostomy. The arteriogram (Fig. 6) was obtained 1 year later. The patient is well 18 months later with good renal function and patent vessels.

\section{Results}

The results are summarized in Table 1 . Ten of the grafts required relatively simple type- 1 or type- 2 reconstruction, but the other 10 needed more sophisticated type- 3 or type- 4 repair. The average time to perform the back table anastomoses was $60 \pm 20 \mathrm{~min}$.

Eighteen of 20 patients transplanted with reconstructed arterial kidneys required postoperative dialysis for acute tubular necrosis (ATN) (90\%), which is significantly higher than the $30 \%$ overall ATN rate observed in the remainder of our cases in 1986. The renal scans performed at 4-6 weeks after transplant showed improved flow in all the surviving kidneys compared to the results during the first few weeks, demonstrating progressive improvement in the vascularization of these kidneys. Anticoagulation was not used postoperatively, but 10 of the 20 patients were given aspirin plus persantin. There were no thrombotic episodes.

Follow-up in December 1987 was 12-24 months, the median being 19 months. Five of the grafts (25\%) were eventually lost after a mean interval of 1 month (range 7 days to 4 months). Irreversible acute or chronic rejection accounted for one loss each of a kidney with type-1, -3, and -4 reconstruction. There was one example after a type-4 reconstruction of primary nonfunction, followed by an infection. Finally, a kidney was lost after a type-2 reconstruction because of acute disruption of the arterial anastomosis secondary to a mycotic infection (Candida albicans) of the aortic patch. The patient underwent an emergency nephrectomy and required ligation of the external iliac artery and a femorofemoral bypass. He underwent successful retransplantation 7 months later.

There was no mortality in these 20 cases. The mean current creatinine of the 15 patients with functioning kidneys is $2.8 \mathrm{mg} \%$ with median follow-up 19 months. The patient with the complex reconstruction shown in Fig. 6 has a creatinine of $2.4 \mathrm{mg} \% 18$ months postoperatively.

\section{Discussion}

The risk of injury to the renal artery during harvesting is high, mainly due to the frequency of multiple vessels in the normal population (more than $20 \%$ of cases). When anomalies are present, the risk is compounded by the haste with which organs are harvested from unstable donors, especially when the donor surgeon is fairly inexperienced.

Methods of renal artery reconstruction for a number of indications have been described previously $[1,5,6,9]$. Our report emphasizes the ex vivo techniques of repair following harvest injury of the renal artery. Comparison with the results obtained with uninjured grafts is also provided after a median follow-up of 19 months.

In 1986 at the University of Pittsburgh, 7.5\% of 270 cadaveric donor kidneys sustained an arterial injury at the time of harvest and underwent back table reconstruction in order to become transplantable. The experience gained in the revascularization of liver grafts [7,11] prompted the use of the same techniques for vascular reconstruction in kidney transplantation, with particular reference to the use of extension iliac allografts. It should be noted that, in addition to the arterial grafts reported here, during the same calendar year we performed approximately 
50 venous reconstructions using extension iliac venous homografts, without any incidence of thrombosis (unpublished observations). Such venous reconstructions were done either because of harvesting injury of the renal vein or the unavailability of the donor cava with the right kidney. These external or common iliac venous homografts were sewn end-to-end to the donor renal vein on the back table, or alternatively, in unusually deep recipient vessels, anastomosed to the recipient iliac vein first, and then to the donor renal vein.

Three questions were asked about the use of these kidneys with repaired arteries.

1. Is there a mortality associated with their use? The answer based on our experience is "no." None of our patients died and the one patient who underwent an emergency nephrectomy because of acute disruption of the anastomosis finally recovered and was retransplanted successfully.

2. Is there any associated morbidity? The answer here is "yes." We observed a significantly higher rate of ATN in these patients (90\%) compared with the nonreconstructed kidney grafts of the same year (30\%). This could be due to lack of perfusion of the graft during and after the harvest and to wastage during the cold flush through the injury. The more complex the arterial repair, the slower the recovery. Special attention should be paid to the case depicted in Fig. 6. This was the most extensive reconstruction known to have been satisfactory.

Rejection of the endothelium has been thought to be a determinant when arterial homografts are used for vascular reconstruction in nonimmunosuppressed patients $[2,3,12]$. In our cases in which iliac extension allografts were used, the immunosuppression required to control the organ rejection may have contributed to the high patency rate. The same effect has been observed after replacement of the hepatic artery with extension iliac allograft in liver transplantation [11].

3. Is it worth it? The answer is "yes." The 1-year graft survival in cases with reconstructed arteries is about 75\%, which is not statistically different from the overall kidney transplant survival obtained with uninjured grafts. The approach has allowed utilization of valuable renal grafts that would otherwise be lost.

In conclusion, injuries to the renal artery during harvesting can be repaired in several ways, utilizing both basic and complex techniques of vascular reconstruction. These repaired kidneys have a long-term success rate that is similar to that after renal transplantation of uninjured grafts, although there is a higher incidence of acute tubular necrosis and delayed functional recovery. The widespread application of such techniques should allow improved utilization of kidneys for transplantation.

\section{Acknowledgments}

We are tremendously grateful to Lauro Truffa for the drawings, Sandi Mitchell for the kidney data registry management, and Donna Ross for typing. This work was supported by research grants from the Veterans Administration and Project Grant No. AM 29961 from the National Institutes of Health, Bethesda, Maryland. V. M. was supported, in part, by the Italian Association for Cancer Research.

\section{References}

1. Dean RH, Meacham PW, Weaver FA. Ex vivo renal artery reconstructions: indications and techniques. J Vasc Surg 1986;4:546-552. [PubMed: 3783829]

2. Deterling RA, Claus RH. Long term fate of aortic and arterial homografts. J Cardiovasc Surg 1970;11:35-43. [PubMed: 5441903] 
3. Galumbeck MA, Sanfilippo FB, Hagen PO, Seaber AV, Urbaniak JR. Inhibition of vessel allograft rejection by endothelial removal. Morphologic and ultrastructural changes. Ann Surg 1987;206:757766. [PubMed: 3500679]

4. Lacombe, M. Surgical techniques in renal transplantation. In: Hamburger, J.; Crosuier, J.; Bach, JF.; Kreis, H., editors. Renal transplantation: theory and practice. 2nd. Williams \& Wilkins; Baltimore: 1981. p. 325-327.

5. Merkel FK, Straus AK, Anderson O, Bannett AD. Microvascular technique for polar artery reconstruction in kidney transplant. Surgery 1976;79:253-261. [PubMed: 769211]

6. Novick AC, Magnusson M, Braun WE. Multiple-artery renal transplantation: emphasis on extracorporeal methods of donor arterial reconstruction. J Urol 1979;122:731-735. [PubMed: 390168]

7. Shaw BW Jr, Iwatsuki S, Starzl TE. Alternative methods of arterialization of the hepatic graft. Surg Gynecol Obstet 1984;159:490-493. [PubMed: 6387980]

8. Simmons, RL.; Najarian, JS. Kidney transplantation. In: Simmons, RL.; Finch, ME.; Ascher, NL.; Najarian, JS., editors. Manual of vascular access organ donation and transplantation. Springer; New York Berlin Heidelberg: 1984. p. 292-309.

9. Starzl, TE. Experience in renal transplantation. Saunders; Philadelphia: 1964. Recipient operation; p. 103-105.

10. Starzl TE, Hakala TR, Shaw BW Jr, Hardesty RL, Rosenthal TE, Griffith BP, Iwatsuki S, Bahnson HT. A flexible procedure for multiple cadaveric organ procurement. Surg Gynecol Obstet 1984;158:223-230. [PubMed: 6367113]

11. Todo S, Makowka L, Tzakis AG, Marsh JW Jr, Karrer FM, Armany M, Miller C, Tallent MB, Esquivel CO, Gordon RD, Iwatsuki S, Starzl TE. Hepatic artery in liver transplantation. Transplant Proc 1987;19:2406-2411. [PubMed: 3547931]

12. Williams GM, Teer-Haar A, Krajewski C, Parks LC, Roth J. Rejection and repair of endothelium in major vessel transplants. Surgery 1975;78:694-706. [PubMed: 1188613] 


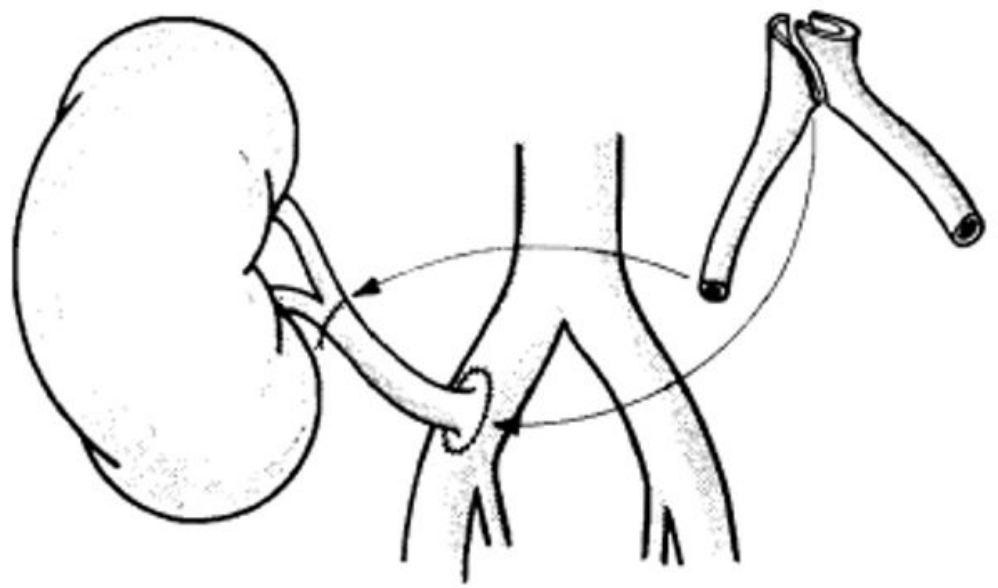

Fig. 1.

Extension of single renal artery using a donor external iliac graft 


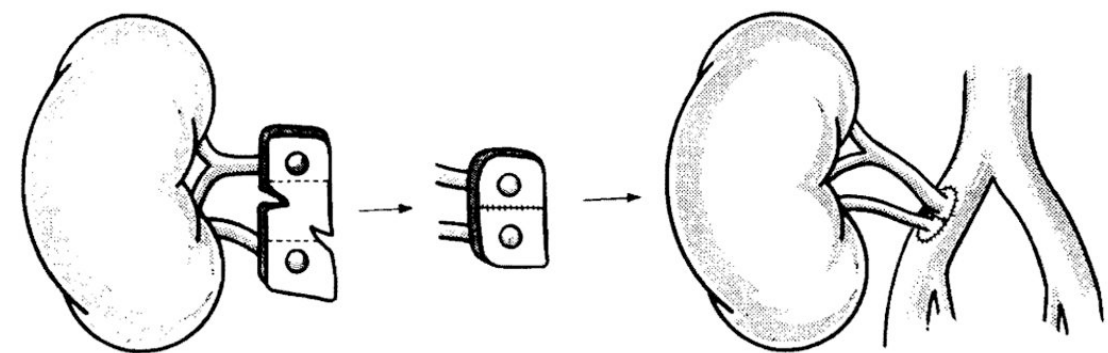

Fig. 2.

Remodeling of the aortic patch in a double-artery kidney 


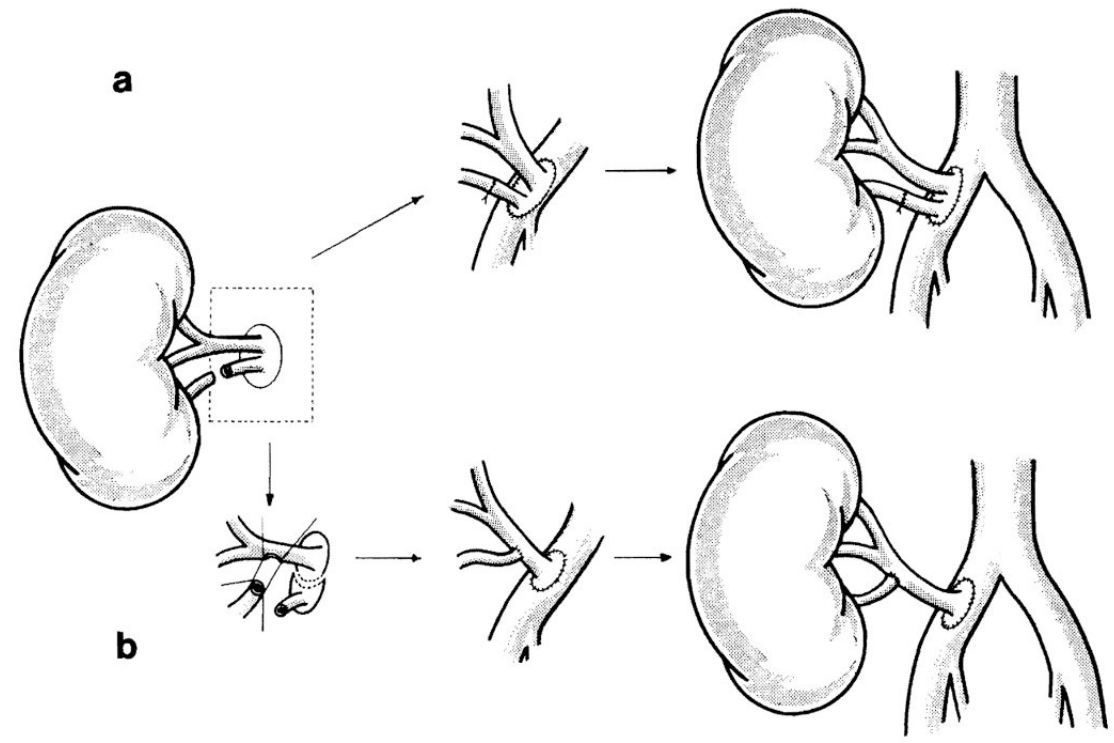

Fig. 3a, b.

Repair of injuries to segmental artery, a Direct repair and $\mathbf{b}$ reimplantation into a larger renal vessel 


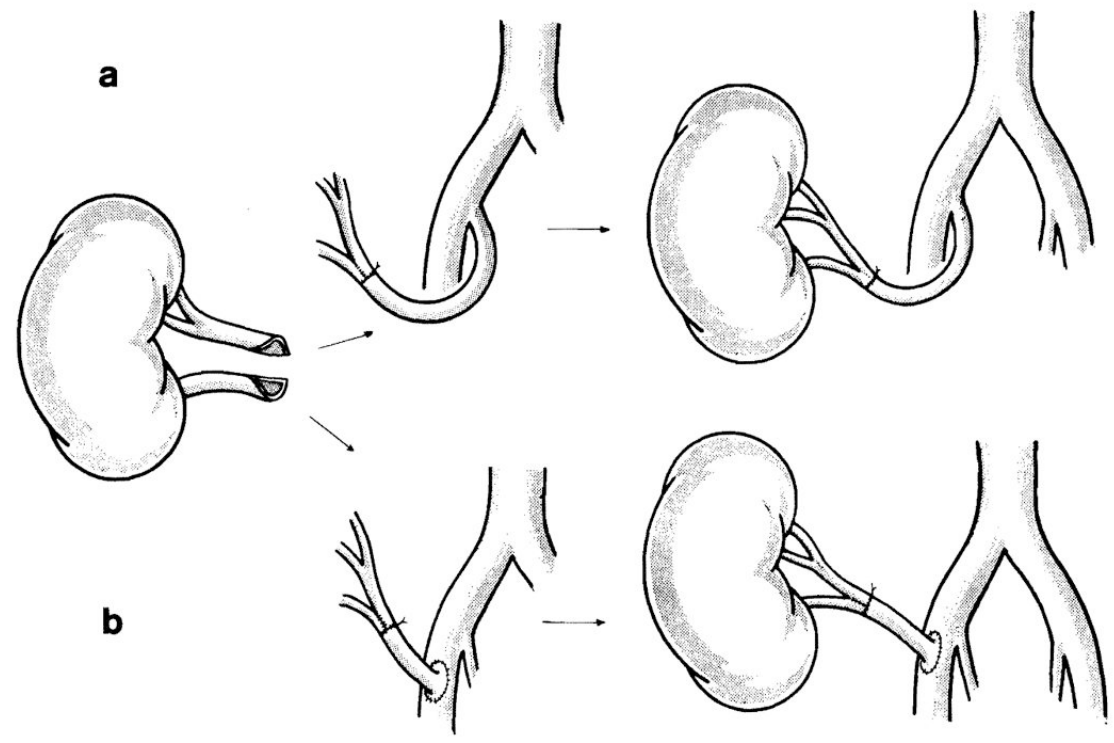

Fig. 4a, b.

Repair of injuries of segmental artery with a side-to-side "conjoined" anastomosis a with the hypogastric artery and $\mathbf{b}$ with a donor vascular graft 

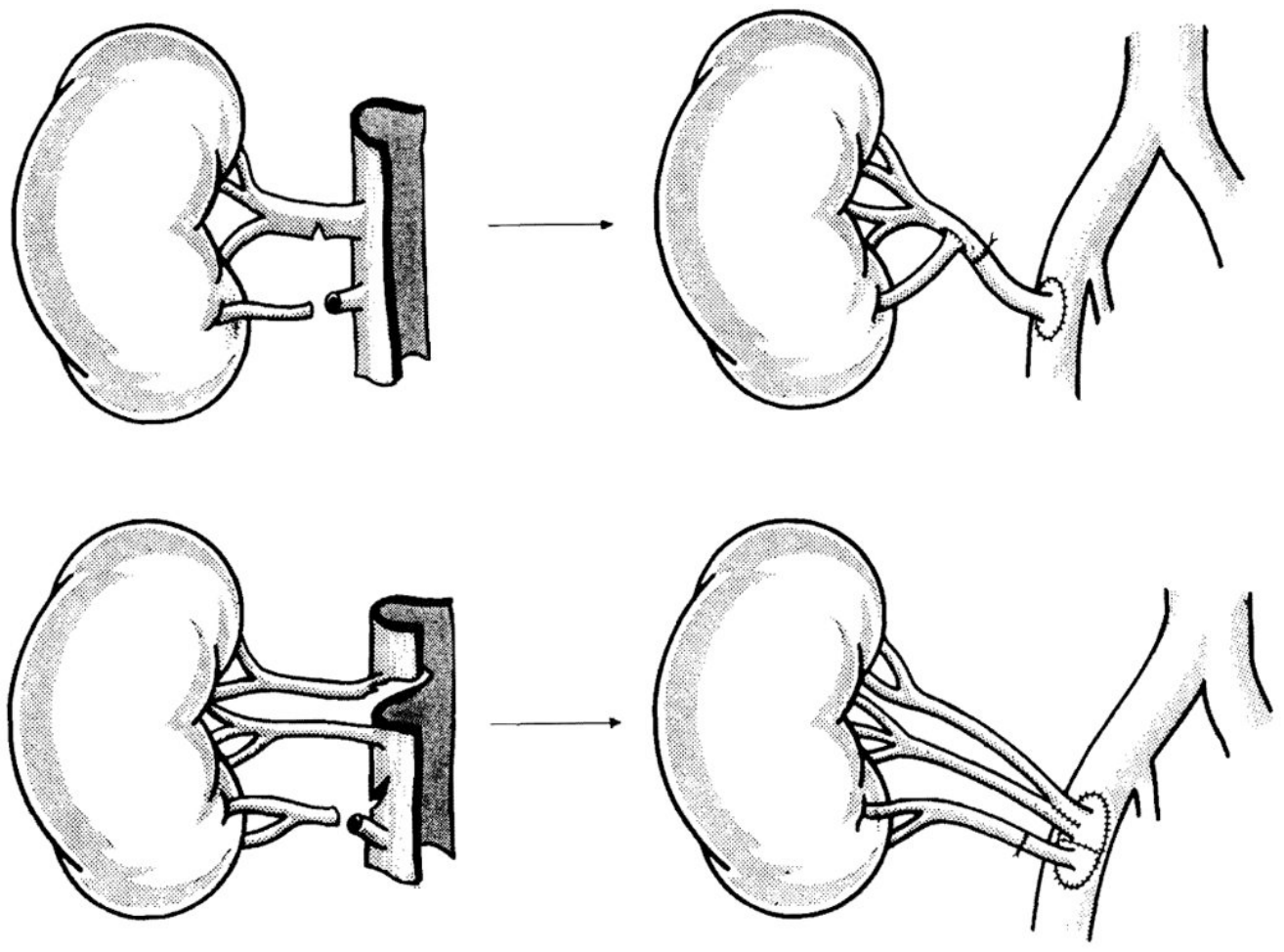

Fig. 5.

Combined reconstruction techniques 

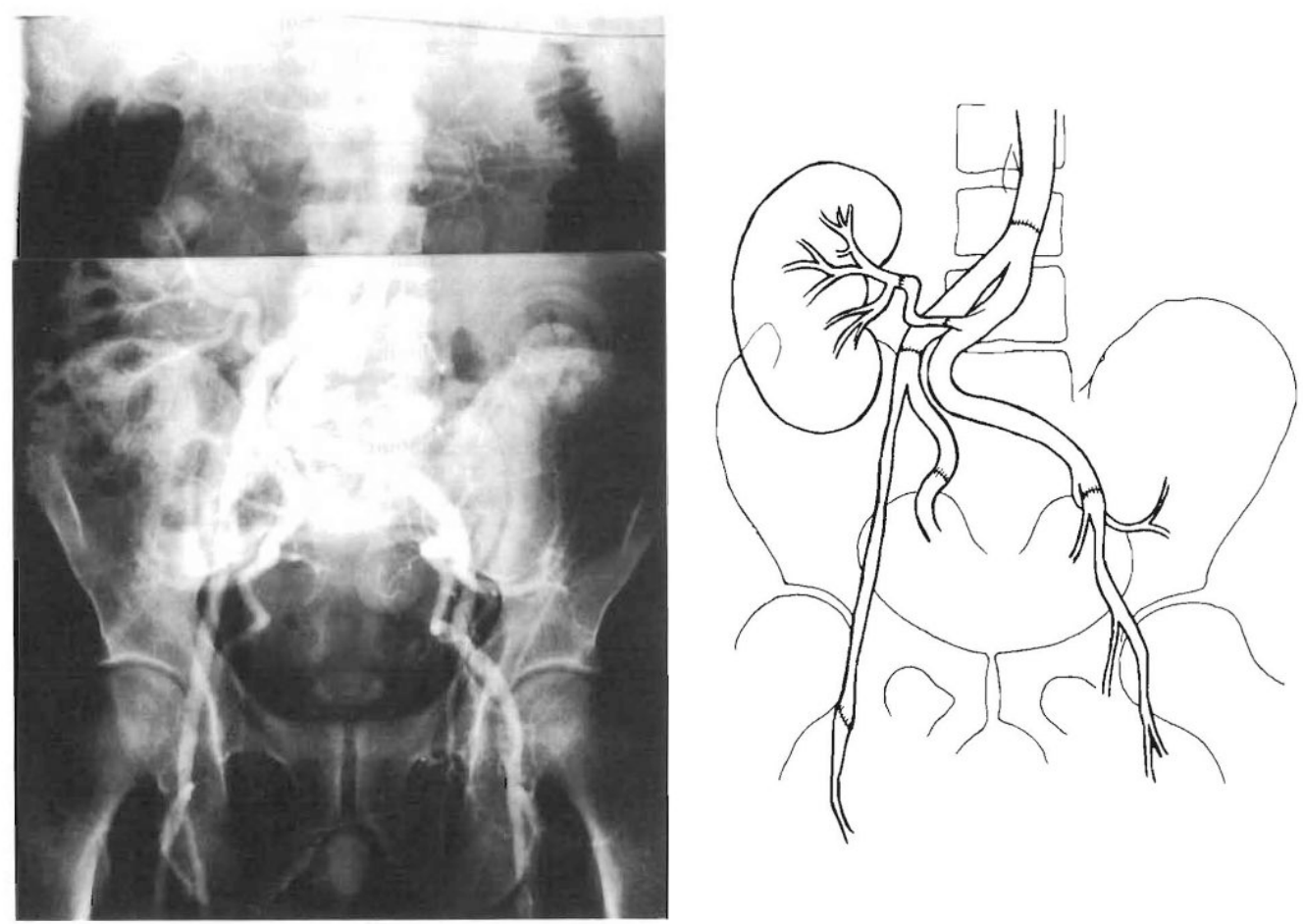

Fig.6.

Extensive arterial reconstruction using aortoiliac homografts. Arteriogram: the first graft replaces the aortic bifurcation, the second bridges the right iliac system, and the third is used to connect the donor renal artery previously injured. In addition, a right femoropopliteal bypass with a reversed autologous saphenous vein graft was needed distally. All anastomoses and vessels are patent 12 months after the kidney transplant. Immunosuppression with cyclosporin and steroid 


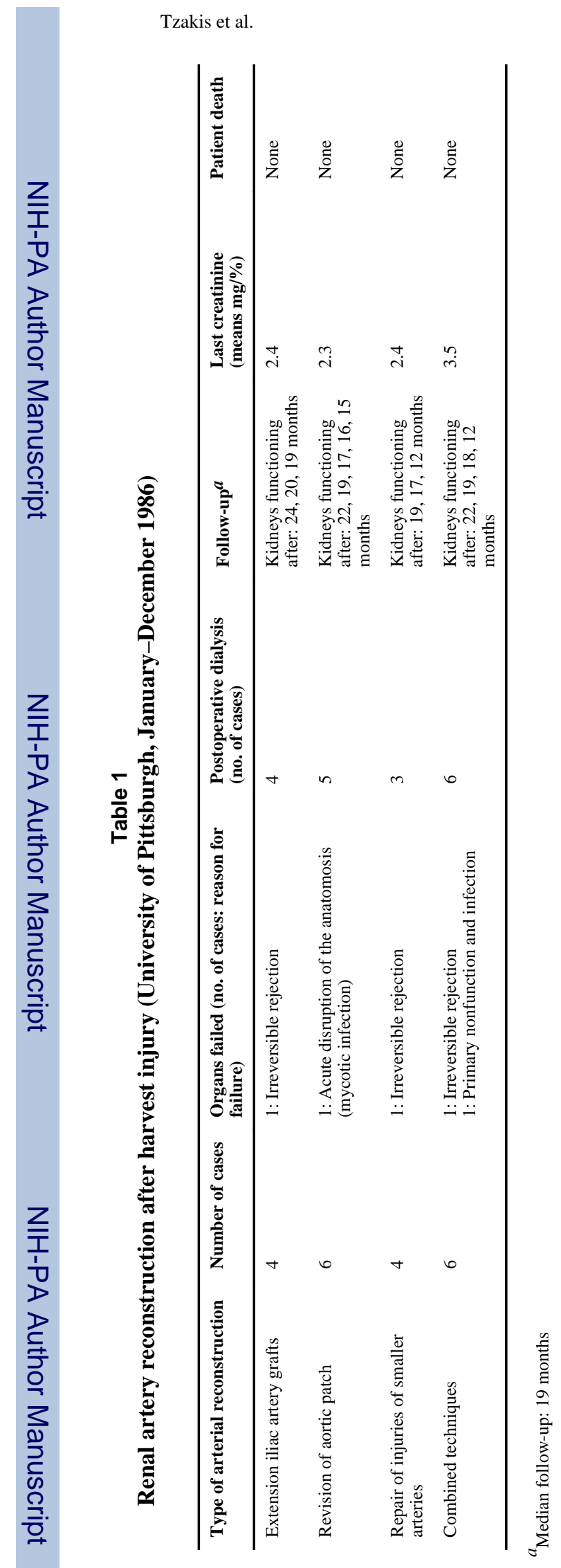

Transpl Int. Author manuscript; available in PMC 2010 November 8. 